ñNew developments on the synthesis of oligonucleotide-peptide conjugatesò Portela, C., Mascareñas, J.L., Albericio, F., Mazzini, S., Caminal, C., Ramos, R., Ocampo, S.M., Eritja, R. Nucleosides, Nucleotides \& Nucleic Acids, 26(8-9), 963-967 (2007). doi: 10.1080/15257770701508216

\title{
New developments in the synthesis of oligonucleotide-peptide conjugates.
}

Cecilia Portela ${ }^{1}$, José Luis Mascareñas ${ }^{1}$, Fernando Albericio ${ }^{2}$, Stefania Mazzini ${ }^{3}$, Clara Caminal $^{2,4}$, Roger Ramos ${ }^{2,4}$, Sandra M. Ocampo ${ }^{2,4}$, Ramon Eritja ${ }^{2,4}$.

${ }^{1}$ Dpto de Química Orgánica. Universidad de Santiago de Compostela, 15782 Santiago de Compostela, Spain.

${ }^{2}$ Institute for Research in Biomedicine (IRB) Barcelona, Parc Científic de Barcelona, Josep Samitier 1-5, 08028 Barcelona, Spain

${ }^{3}$ DISMA, Universita degli Studi di Milano. Via Celoria 2, I-20133 Milano. Italy. ${ }^{4}$ Instituto de Biologia Molecular de Barcelona. C.S.I.C., Jordi Girona 18-26, 08034 Barcelona, Spain

\section{Running title: oligonucleotide-peptide conjugates}

Abstract. The stability of oligodeoxynucleotides to trifluoroacetic acid is studied. Pyrimidine oligonucleotides were stable in the conditions used for the removal of $t$ butyl groups. Oligonucleotide-3ôpeptide conjugates carrying pyrimidine oligonucleotides are prepared stepwise using peptide-supports and Fmoc, $t$-butyl strategy. Using this strategy we have prepared an oligonucleotide-peptide conjugate containing as peptide the leucine-rich fragment of FOS, a transcription factor involved in many important cellular processes. This conjugate has a long peptide sequence with a large number of trifunctional amino acids. 
Introduction. The use of synthetic oligonucleotides to control gene expression has triggered the search for new oligonucleotide derivatives with improved therapeutic potential $^{[1]}$. Oligonucleotide-peptide conjugates are chimeric molecules consisting of oligonucleotides covalently linked to peptides. As a result, synthetic oligonucleotides acquire some of the biological and/or biophysical properties of peptides.

Two strategies can be followed to synthesize oligonucleotide-peptide conjugates $^{[2]}$. In the post-synthetic conjugation approach, the two moieties are prepared independently and specific groups (such as thiols and maleimido) are specifically incorporated to link both molecules ${ }^{[3]}$. In the stepwise approach, oligonucleotide-peptide conjugates are prepared by stepwise addition of amino acids and phosphoramidites in solid phase on the same solid support ${ }^{[4]}$. In this case, the problem is the incompatibility of the standard schemes of protection for peptides and oligonucleotides. For example, at the end of the solid-phase peptide synthesis, a treatment with acid is usually required, which can provoke partial depurination of DNA.

Recently, our group has undertaken the preparation of conjugates consisting of doublestranded DNA (dsDNA) and selected fragments of biologically relevant transcription factors. Specifically we focused on the synthesis of conjugates between DNA and peptidic regions from the leucine zipper domain of $\mathrm{FOS}^{[5]}$. These constructs, which display a dual recognition surface provided by the presence of both the peptide and the DNA, were devised as potential specific receptors for selected FOS partners, such as the well known oncogenic transcription factor JUN. Hypothetically, the recognition would involve a specific interaction between the leucine-rich region of JUN and the hybrid, and an additional interaction between the oligonucleotide portion of the constructs and the basic region of $\mathrm{JUN}^{[6]}$. The synthesis of these conjugates is difficult 
due to the presence of long peptide sequences with large number of trifunctional amino acids. In any case we evaluated the viability of making this type of oligonucleotidepeptide conjugates using a stepwise Fmoc, $t$-butyl strategy.

\section{Results and Discussion.}

Stability of oligodeoxynucleotides to trifluoroacetic acid. Trifluoroacetic acid (TFA) is a strong acid commonly used in the final deprotection step of peptide synthesis using Fmoc-amino acids. It is known that acidic solutions provoke depurination of purine nucleosides. On the other hand, depurination is slower in oligonucleotides and indeed several experiments on the use of TFA to remove Boc groups in the presence of oligodeoxynucleotides were described almost two decades ago ${ }^{[7,8]}$. In order to study the extension of this reaction we have treated 4 oligodeoxynucleotide sequences $\left(\mathrm{T}_{9}, \mathrm{~T}_{4} \mathrm{CT}_{4}\right.$, $\mathrm{T}_{4} \mathrm{AT}_{4}$ and $\mathrm{T}_{4} \mathrm{GT}_{4}$ ) with $95 \%$ TFA aqueous solution. Two different treatments were studied. Treatment A: The oligonucleotide supports (polystyrene, LV200) obtained after the assembly of the oligonucleotide sequence still carrying the protecting groups were treated with 95\% TFA aqueous solution. After 2 hours, the acidic solution was filtered out and the support was treated with concentrated ammonia. The resulting solution was concentrated to dryness and analyzed by reversed-phase HPLC. Treatment B: Oligonucleotides were deprotected with ammonia and the resulting unprotected oligonucleotides were treated with $95 \%$ TFA aqueous solution for 2 hours. The resulting solutions were concentrated to dryness, treated with ammonia (to break apurinic sites) and analyzed by HPLC.

Pyrimidine oligonucleotides $\left(\mathrm{T}_{9}\right.$ and $\left.\mathrm{T}_{4} \mathrm{CT}_{4}\right)$, after treatment $\mathrm{A}$, gave a single peak with the expected mass by MALDI MS, indicating stability of the oligonucleotides to the 
TFA treatment as it is expected due to the higher stability of the glycosidic bond of pyrimidine nucleosides. Exceptionally, treatment $\mathrm{B}$ on $\mathrm{T}_{4} \mathrm{CT}_{4}$ with TFA promoted a slight decomposition. Oligonucleotide sequence carrying adenine $\left(\mathrm{T}_{4} \mathrm{AT}_{4}\right)$ gave two more peaks eluting before the peak of the initial oligonucleotide (Figure 1). This indicates loss of the adenine followed by hydrolysis of phosphate at the abasic site by ammonia. The extension of the depurination was around $80 \%$ in treatment $\mathrm{A}$ and $60 \%$ in treatment B. Oligonucleotide sequence carrying guanine $\left(\mathrm{T}_{4} \mathrm{GT}_{4}\right)$ was stable to treatment A but unstable to treatment $\mathrm{B}$ ( $30 \%$ depurination). This is consistent with the higher stability of dG to depurination compared to dA. Depurination was more severe in treatment B probably because in solution reactions are faster and because of the additional time of exposure to acids during concentration of the solution.

Synthesis of pyrimidine oligonucleotide conjugates carrying the leucine zipper domain of FOS. The stability of pyrimidine oligodeoxynucleotides to TFA suggest that pyrimidine oligonucleotide conjugates may be prepared by addition of the oligonucleotide sequence to the peptide support carrying the leucine zipper domain of FOS assembled with the standard Fmoc, $t$ Bu-strategy. Scheme 1 outlines the synthetic approach. The peptide part is a peptide of 35 amino acids carrying 25 trifunctional amino acids, two of which are arginines. The oligonucleotide moiety is tridecathymidine.

The synthesis of the 35 amino acid peptide was carried out manually on a RinkMBHA amide resin using Fmoc-protected amino acids and a symmetric anhydride strategy. In order to incorporate the oligonucleotide sequence to the peptide support, the amino terminal group was reacted with the O-4-trityl derivative of 4hydroxybutanoic acid. This compound will generate the hydroxyl group which will be 
used to assemble the oligonucleotide sequence (Scheme 1). Figure 2 shows the HPLC analysis of the $\mathrm{T}_{13}$-leucine zipper domain conjugate (Waters X-Terra column at a flow rate of $1 \mathrm{~mL} / \mathrm{min}$, detection $260 \mathrm{~nm}$, gradient $15-80 \% \mathrm{~B}$ in $20 \mathrm{~min}, \mathrm{~A}: 5 \% \mathrm{CH}_{3} \mathrm{CN}$ in $100 \mathrm{mM}$ TEAA $\mathrm{pH}$ 7.0, B: $70 \% \mathrm{CH}_{3} \mathrm{CN}$ in $100 \mathrm{mM}$ TEAA $\mathrm{pH}$ 7.0). A major peak corresponding to the desired conjugate was observed and characterized by UV, PAGE and MALDI MS. To the best of our knowledge these conjugates are among the largest and more complex oligonucleotide-peptide conjugates constructed to date by chemical synthesis, and in particular by solid-supported stepwise methods.

Project financed by Spanish Ministry of Education SAF2004-01044, BFU2005-23719E, NAN2004-09415-C05-03 and BFU2004-02048. C. P. thanks the Spanish Ministry for a predoctoral fellowship.

\section{References}

1. Gait, M.J. 2003. Peptide-mediated cellular delivery of antisense oligonucleotides and their analogues. Cell. Mol. Life Sci., 60, 844-853

2. Tung, C.H., and Stein S. 2000. Preparation and applications of peptideoligonucleotide conjugates. Bioconjugate Chem., 11, 605-618.

3. Eritja, R., Pons, A., Escarceller, M., Giralt, E., and Albericio, F. 1991. Synthesis of defined peptide-oligonucleotide hybrids containing a nuclear transport signal sequence. Tetrahedron, 47, 4113-4120.

4. Grandas, A., Marchan, V. Debéthune, L., and Pedroso, E. 2004. Stepwise solidphase synthesis of nucleopeptides in Current protocols in Nucleic Acid Chemistry; John Willey, New York chapter 4.22.1. 
5. Glover, J.N., and Harrison, S.C. 1995. Crystal structure of the heterodimeric bZIP transcription factor c-Fos-C-Jun bound to DNA. Nature, 373, 257-261.

6. Olive, M., Krylov, D., Echlin, D.R., Gardner, K., Taparowsky, E. and Vinson, C. 1997. A dominant negative to activation protein-1 (AP1) that abolishes DNA binding and inhibits oncogenesis. J. Biol. Chem., 272, 18586-18594.

7. Haralambidis, J., Duncan, J. and Tregear, G.W. 1987.The solid phase synthesis of oligonucleotides containing a 3ôpeptide moiety. Tetrahedron Lett., 28, 51995202.

8. Haralambidis, J., Chai, M. and Tregear, G.W. 1987. Preparation of basemodified nucleosides suitable for non-radioactive label attachment and their incorporation into synthetic oligonucleotides. Nucleic Acids Res., 15, 48574876. 


\section{FIGURES AND SCHEMES}

Scheme 1. Outline of the synthesis of oligonucleotide conjugate $\mathrm{T}_{13}$-leucine zipper domain by the stepwise approach.
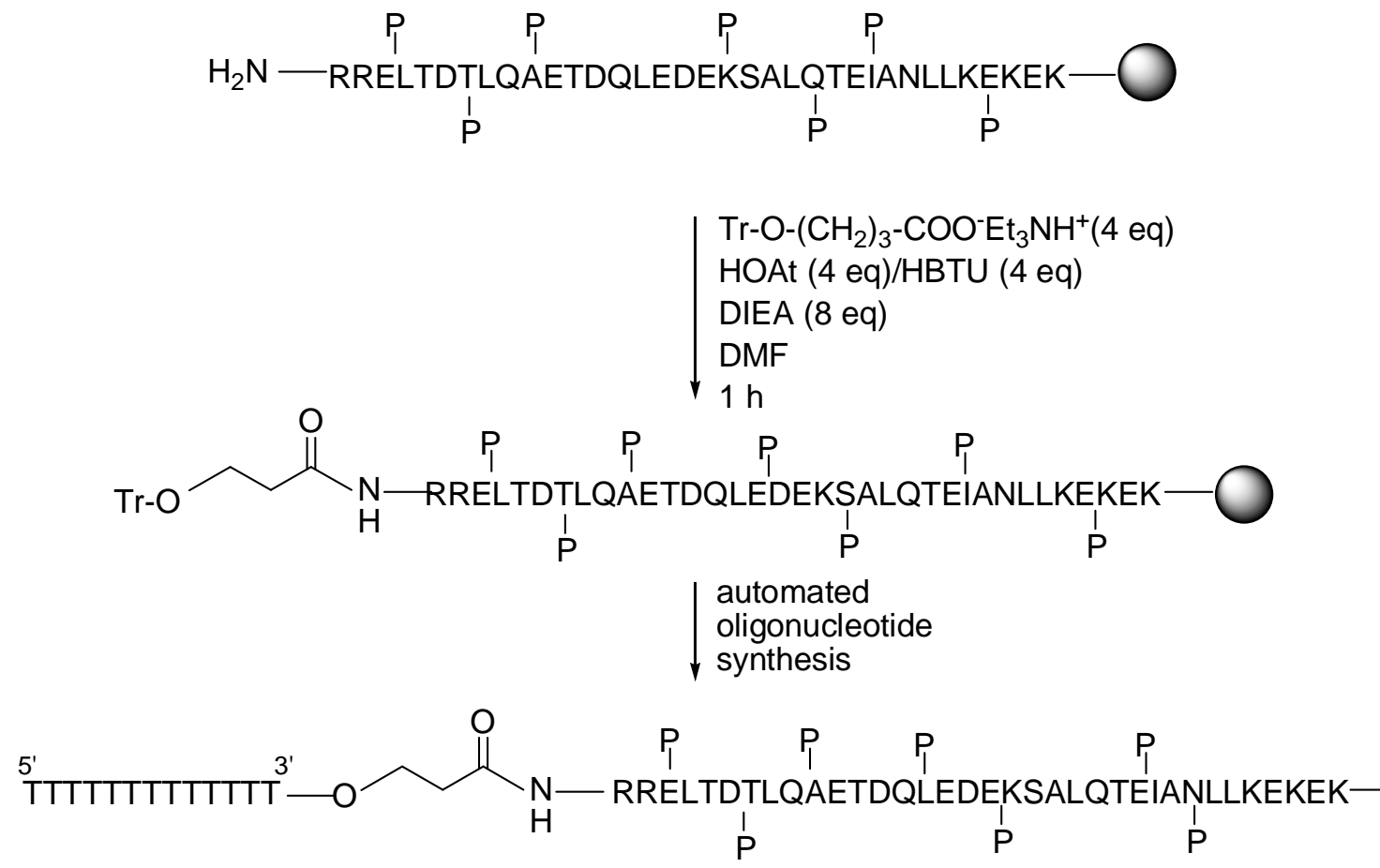

1. $20 \%$ piperidine/DMF

2. $\mathrm{TFA} / \mathrm{H}_{2} \mathrm{O}(95: 5)$

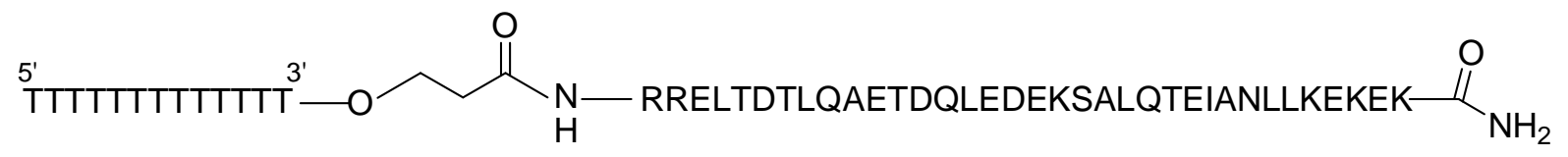


Figure 1. HPLC of the mixture resulting from the treatment of the support carrying $\mathrm{T}_{4} \mathrm{AT}_{4}$ followed by ammonia deprotection. The late-eluting peak is the remaining fulllength oligonucleotide sequence $\mathrm{T}_{4} \mathrm{AT}_{4}$. HPLC conditions as follows. Nucleosil 120 $\mathrm{C} 18(250 \times 8 \mathrm{mmm})$ column at a flow rate of $3 \mathrm{~mL} / \mathrm{min}$, detection $260 \mathrm{~nm}$, gradient 0 $50 \% \mathrm{~B}$ in $20 \mathrm{~min}, \mathrm{~A}: 5 \% \mathrm{CH}_{3} \mathrm{CN}$ in $100 \mathrm{mM}$ triethylammonium acetate (TEAA) $\mathrm{pH}$ 7.0, B: $70 \% \mathrm{CH}_{3} \mathrm{CN}$ in $100 \mathrm{mM}$ TEAA $\mathrm{pH}$ 7.0.

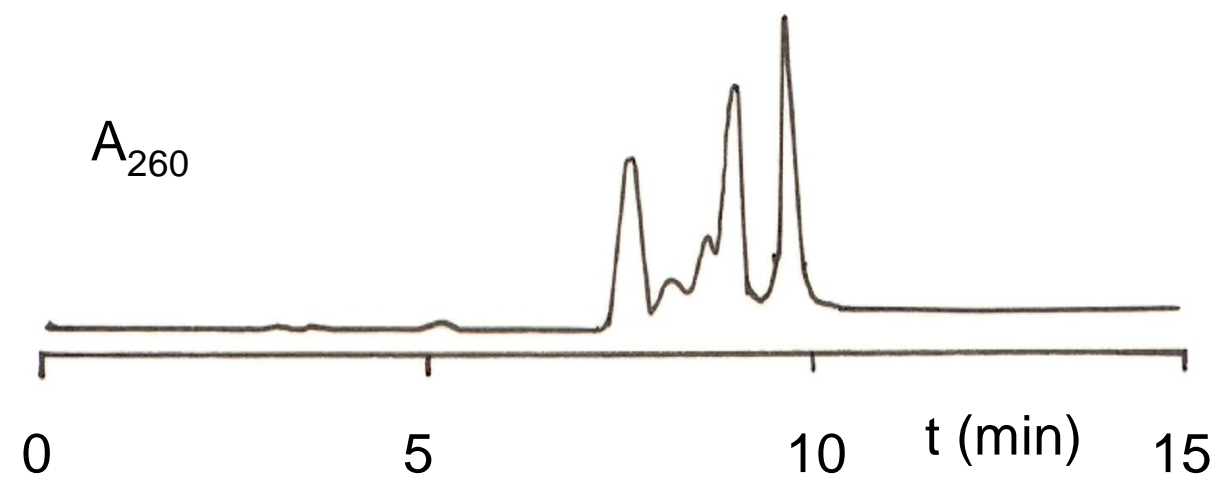

Figure 2. HPLC purification of oligonucleotide conjugate $\mathrm{T}_{13}$-leucine zipper domain. The peak at 10.9 min is the expected oligonucleotide conjugate. HPLC conditions in the insert.

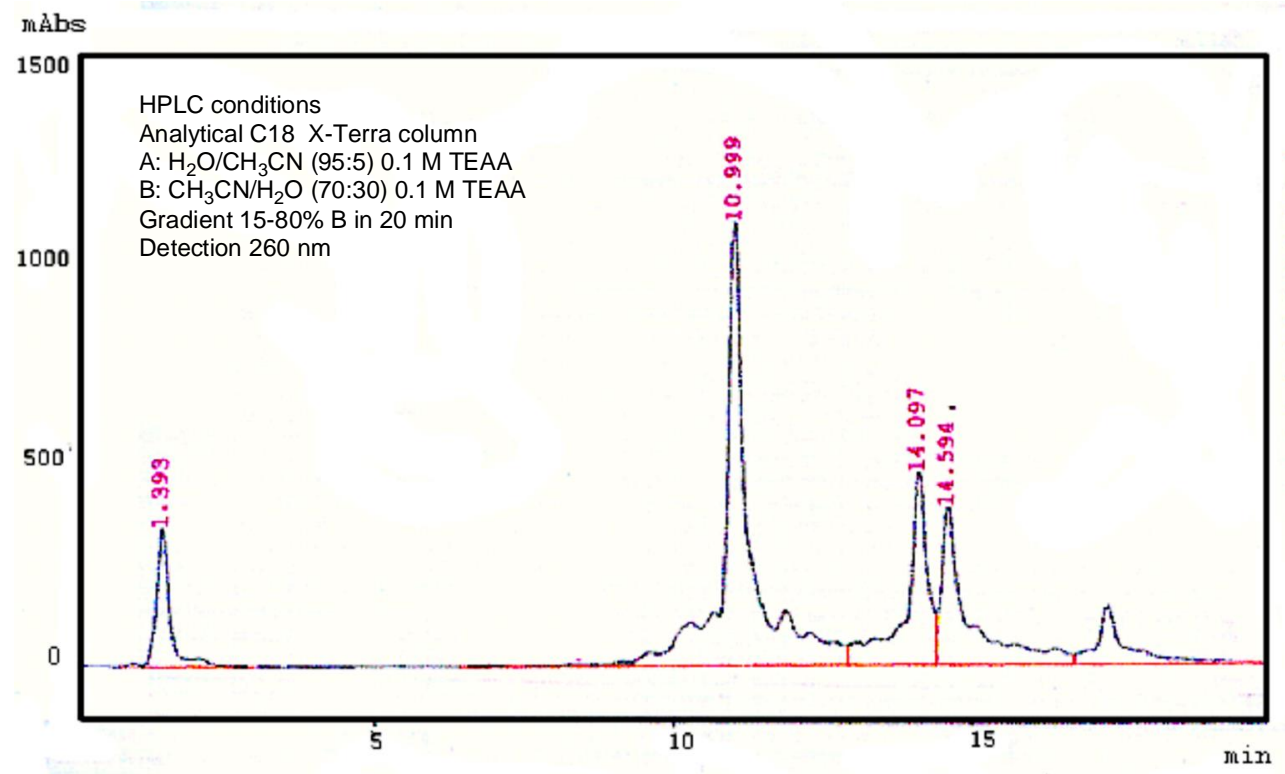

